La revue La revue pour l'histoire du CNRS

POUR L'HISTOIRE DU CNRS $\quad 15 \mid 2006$

CNRS et Université

\title{
Passerelle des sciences : la contractualisation tripartite
}

Bernard Bigot

\section{(2) OpenEdition}

Journals

Édition électronique

URL : https://journals.openedition.org/histoire-cnrs/501

DOI : 10.4000/histoire-cnrs.501

ISSN : 1955-2408

Éditeur

CNRS Éditions

Édition imprimée

Date de publication : 3 novembre 2006

ISBN : 978-2-271-06452-3

ISSN : $1298-9800$

\section{Référence électronique}

Bernard Bigot, «Passerelle des sciences : la contractualisation tripartite », La revue pour l'histoire du CNRS [En ligne], 15 | 2006, mis en ligne le 23 novembre 2008, consulté le 20 mai 2021. URL : http:// journals.openedition.org/histoire-cnrs/501; DOI : https://doi.org/10.4000/histoire-cnrs.501

Ce document a été généré automatiquement le 20 mai 2021.

Comité pour l'histoire du CNRS 


\title{
Passerelle des sciences : la contractualisation tripartite
}

\author{
Bernard Bigot
}

1 La base du dispositif de contractualisation aujourd'hui est un contrat quadriennal tripartite entre le ministère de l'Éducation nationale, l'enseignement supérieur et de la recherche, le CNRS et les établissements d'enseignement supérieur et de recherche. Ce contrat engage les signataires sur un budget et des modalités de mise en œuvre, mais surtout sur un programme scientifique clair. Le critère scientifique est déterminant : préalablement à l'établissement du contrat, les laboratoires concernés doivent être évalués par le Comité national de la recherche scientifique et la Mission scientifique du ministère. Plusieurs typologies d'unités de recherche sont concernées, de manière à permettre de traduire, à différents niveaux d'intensité et d'implication, l'engagement partenarial des universités ou des grandes écoles avec le CNRS. Cette mesure a permis de simplifier le travail des laboratoires qui devaient auparavant remplir deux documents différents et souvent redondants.

2 Le bilan est positif. Cette politique de contractualisation conduit à une meilleure cohérence du système et à une bonne connaissance du paysage des laboratoires. Elle offre également à ces derniers une certaine stabilité, en termes de statut comme de moyens, puisqu'elle assure une "visibilité » à quatre ans. Au terme d'une telle période d'activité, une nouvelle évaluation sera effectuée pour chaque unité, dans le cadre du projet quadriennal de l'établissement auquel elle est rattachée.

3 Depuis les années 50, période charnière du développement de la recherche publique en France, l'originalité du dispositif français, par comparaison avec les autres grands pays scientifiques, est sa diversité institutionnelle: plus de 80 universités, 120 grandes écoles, 20 organismes nationaux généralistes ou spécialisés... En revanche, il n'existe pas d'agence nationale de financement, hors ministères, à laquelle leurs équipes pourraient s'adresser pour soutenir certains de leurs projets, au-delà des ressources que leur institution peut directement leur apporter, année après année. Aux yeux de beaucoup, au printemps 1993, au moment où François Fillon prend la tête du ministère 
de l'Enseignement supérieur et de la Recherche, la coopération inter institutionnelle en réponse à ce constat n'était guère satisfaisante. Elle appelait une réflexion approfondie.

C'est dans ce contexte que le ministre lançait en juin 1993 une consultation nationale sur la recherche dont une des conclusions les plus marquantes, formalisée dans un rapport débattu devant le parlement fin juin 1994, était la reconnaissance par l'ensemble des communautés scientifique, économique et politique nationales que cette diversité devait être préservée, mais qu'elle devait être mieux gérée pour pouvoir conserver le dynamisme et l'efficacité de notre recherche.

Le directeur général du CNRS, Guy Aubert, nommé le 19 juillet 1994, initie alors une réflexion au sein du CNRS pour que soient proposées, tant au niveau de son organisation interne qu'au plan de ses relations avec les universités, des réponses aux questions soulevées par la consultation nationale. La Mission scientifique et technique (MST) chargée de l'évaluation de la politique de recherche des universités au sein du ministère suit attentivement l'avancement de ces travaux, en liaison avec la Direction générale de la recherche et de la technologie (DGRT).

Depuis sa création en 1939, l'évolution du CNRS a été indissolublement liée à celle de la recherche universitaire. En 1966, une formalisation de ces relations avait été engagée avec la création des premières Unités de recherche associées (URA): une reconnaissance indispensable pour que le CNRS affecte des personnels dans ces unités implantées au sein des universités. Quinze ans plus tard, se développait le concept d'Unités mixtes de recherche (UMR), développées cette fois-ci avec les entreprises ou d'autres organismes: un partenariat renforcé sur des bases programmatiques scientifiques et financières pluriannuelles. À la fin des années 80 , une extension du concept était retenue pour quelques unités universitaires, mais dans la plupart des cas sans engagement financier pluriannuel de la part du CNRS. De son côté, le ministère chargé de l'Enseignement supérieur avait, à partir de 1990, mis en place des contrats de recherche d'une durée de quatre ans avec les établissements d'enseignement supérieur. Ils assuraient le financement de leurs unités de recherche reconnues, dont leurs UMR et URA. Ces contrats avaient constitué un réel progrès.

7 À l'été 1994, on recensait 238 Unités propres du CNRS (UPR) et assimilées, 135 UMR et 1 107 URA et assimilées. Soient au total 10700 chercheurs CNRS, 16750 professeurs et maîtres de conférences universitaires, 22700 ingénieurs, techniciens et personnels administratifs du CNRS et quelque 5000 personnels équivalents des universités. Les UMR et URA, localisées dans 80 universités et 110 grandes écoles, accueillaient 5850 chercheurs du CNRS alors que les UPR CNRS accueillaient 1400 chercheurs universitaires.

Dans une note d'étape destinée au ministre datée du 12 octobre 1994, en tant que directeur de la MST, je tirais un premier bilan et formulais une "Proposition d'organisation du partenariat CNRS/recherche universitaire ». Il suggérait que le CNRS réorganise "ses structures internes et ses modes de collaborations avec les autres partenaires du dispositif national de recherche. L'objectif est de faciliter l'élaboration et l'expression d'une politique scientifique claire et ambitieuse pour l'organisme, de valoriser et de soutenir le dynamisme scientifique de ses personnels et de renforcer l'intensité et la qualité de ses collaborations avec les établissements d'enseignement supérieur, les autres organismes de recherche publique nationaux ou étrangers, et les entreprises. [...] Le moyen retenu pour engager cette évolution est un accroissement très significatif de la part des moyens du CNRS attribués dans le cadre de programmes 
pluriannuels. [...] Les moyens correspondants seront des moyens consolidés (moyens financiers et moyens en personnel) distribués selon une procédure propre à chaque programme. »

9 La conséquence de cette organisation aboutirait à une clarification de la définition des différentes unités de recherche du CNRS et des universités. Ces unités appartiendraient désormais à trois grandes catégories :

10 - les structures propres du CNRS ;

11 - les unités propres des établissements d'enseignement supérieur ;

12 - les unités mixtes résultant d'un accord contractuel entre le CNRS, un établissement d'enseignement supérieur et le ministère de l'Enseignement supérieur et de la Recherche (MESR), ou d'autres partenaires.

13 La note du 12 octobre précisait: "Étant donné la prise conjointe de décision concernant les UMR, la DGRT/MST, le CNRS et l'établissement d'enseignement supérieur concerné procéderaient la même année et ensemble aux évaluations et aux négociations pour les parties en coresponsabilité dans des contrats quadriennaux. La partie commune serait signée par les trois parties. »

14 Tout s'accélérait ensuite à l'automne 1994. Le 14 octobre, le chef de la MST présentait devant le bureau de la Conférence des présidents d'universités (CPU), puis devant celui de la Conférence des directeurs d'écoles et formations d'ingénieurs (CDEFI), les orientations proposées par le CNRS et le ministère. Il précisait que ce schéma était ouvert à une large discussion avant toute mise en œuvre. En réponse, lors d'une rencontre avec le ministre, la CPU indiquait que «l'idée de contractualisation CNRS/ université/MESR passe plutôt bien, mais [qu']il faut veiller à ce que la répartition de moyens soit clairement définie à cette occasion ». La suite se déroulait en quelques jours. Premier temps, les présidents des sections du Comité national de la recherche scientifique signalaient au chef de la MST qu'ils «sont préoccupés par les projets de restructuration du CNRS. Ils demandent à être consultés dans le cadre de la concertation engagée ». Deuxième temps, le ministre demandait "aux universités d'examiner, dans un esprit de dialogue, les propositions conjointes du CNRS et des services du ministère », en soulignant qu'il convenait "effectivement de débattre des principes du projet d'évolution des relations CNRS-universités comme des modalités pratiques de sa mise en œuvre ». Troisième temps, le 28 octobre, la CDEFI adressait au ministre une note dans laquelle elle indique «le caractère positif d'une démarche visant à responsabiliser les établissements universitaires dans leur politique de recherche à condition que ces établissements disposent d'une réelle autonomie assortie d'un contrôle a posteriori ».

15 Le président de la Commission recherche de la CPU écrivait lui aussi au ministre, le 16 novembre, que l'étape de discussions entre la CPU et le ministère " est positivement vécue». "L'idée de contrat et de véritable partenariat avec budget consolidé est intéressante et constitue une vraie nouveauté en ce sens qu'elle impose aux établissements la nécessité de politiques scientifiques et de formations cohérentes et reconnues dans un contrat tripartite », notait-il. Conclusion : « la réforme proposée par le CNRS doit nécessairement contribuer à un renforcement de l'ensemble de la recherche française et non pas avoir un effet de déstructuration de la recherche universitaire. " Les interrogations portaient essentiellement sur les transferts de charge. 
16 Ces débats, primordiaux, se déroulaient dans un contexte tendu en raison des difficultés financières du CNRS. Ayant sur engagé en 1990-1993 les ressources dont il disposait, le CNRS terminait l'année 1993 avec environ 500 MF de factures qu'il n'était pas en mesure de payer. Guy Aubert constatait dès son arrivée que la direction précédente avait notifié aux laboratoires la totalité les autorisations de programmes disponibles, sans qu'une explication soit donnée aux directeurs des laboratoires sur la nature de ces notifications. Or, l'écart entre les autorisations de programmes et les crédits de paiements services votés par le Parlement avait été depuis 1992 de 550 MF au total. Cette situation était compliquée par le fait qu'il y avait au niveau des laboratoires confusion des crédits sur subvention d'État et sur ressources associées à des contrats avec des tiers (entreprises, collectivités, Europe). Le nouveau directeur général a alors lancé une opération de clarification qui conduisait à la suspension de toute commande des laboratoires, sur crédits CNRS, au mois de septembre 1994 et un droit de consommation, en moyenne, des autorisations de crédits affectés aux laboratoires limité à 60 \% au titre de l'année 1994.

On imagine aisément les tensions que ces décisions créaient immédiatement au sein du CNRS et les protestations et manifestations, pour certaines légitimes, pour d'autres sans autre justification que celle de l'agitation à l'approche des élections présidentielles de 1995. Un article de la revue Nature du 20 octobre expliquait, sans aucun fondement : «la protestation des chercheurs la semaine dernière est entretenue par une circulaire gouvernementale non publiée qui conduirait à réduire le nombre de départements scientifiques du CNRS de sept à trois et ses laboratoires de 1300 à 400, avec le contrôle de nombreux laboratoires passant directement aux universités ». Le 28 novembre, un autre article (Libération) s'intéressait aux propositions d'évolution des relations CNRSuniversités: "un premier scénario conduirait à s'attaquer enfin aux problèmes structurels de fond générés par des décennies de dérives institutionnelles et gestionnaires. Une redéfinition de ce qui est attendu des universités et des universitaires en matière de recherche [....] est inévitable, mais en fait [l'objectif de la réforme] est le démantèlement [du CNRS] et le retour à [son] asservissement au mandarinat universitaire le plus réactionnaire qui soit. "

$18 \mathrm{Au}$ mois de décembre, le ministre écrivait à Guy Aubert. "Intensifier et clarifier le partenariat entre le CNRS et les établissements d'enseignement supérieur est pour moi une priorité, écrivait François Fillon. [...] Des contrats seront mis en place dès cette année entre l'État et les établissements d'enseignement supérieur. Je souhaite que ces contrats couvrent tous les aspects de leur fonctionnement, et en particulier la recherche. Ils constituent, [dans le cadre d'une véritable négociation], un instrument bien adapté pour améliorer le partenariat entre l'État, le CNRS et la recherche universitaire [...] Je souhaite voir un grand nombre d'établissements s'engager dans cette démarche pour l'heure expérimentale et fondée sur le volontariat. Un premier bilan sera réalisé à l'automne 1995 afin d'examiner l'opportunité et les modalités de son éventuelle généralisation ».

19 L'année 1995 voyait l'achèvement de la réforme. Dès le mois de janvier, le CNRS et les services du ministère transmettaient au ministre un document sur le « renforcement $\mathrm{du}$ partenariat CNRS/recherche universitaire ». Reprenant les réflexions d'octobre 1994, il proposait que les établissements des académies de Bordeaux, Toulouse, Montpellier, Grenoble, Lyon et Dijon, concernés par la campagne de contractualisation lancée par le MESR, puissent entrer dès 1995 dans cette logique d'une négociation 
contractuelle tripartite. Le 27 janvier 1995, le ministre confirmait aux présidents des universités et directeurs d'écoles qu'il était en accord avec ces propositions. Au printemps 1995, l'ensemble des établissements concernés, à l'exception d'un seul, avait donné son accord pour s'engager dans cette négociation. Celle-ci aboutissait à la signature de plus de 45 contrats tripartites dès la fin 1995 avec la création de nombreuses UMR, essentiellement par transformation d'anciennes URA. Cette procédure s'est généralisée à l'ensemble des universités et fonctionne jusqu'à ce jour, même si l'inflation du nombre des UMR sous diverses pressions a quelque peu altéré son efficacité. De l'avis de tous, elle a incontestablement constitué un progrès dans les relations CNRS-universités.

Elle ne représente cependant qu'une étape. La nouvelle loi sur la recherche publiée début 2006, d'une certaine manière, prolonge ce mécanisme en l'actualisant, pour répondre à l'évolution du contexte universitaire et de la recherche dans le monde.

\section{RÉSUMÉS}

Depuis une trentaine d'années, le CNRS et l'enseignement supérieur sont liés au sein des laboratoires qu'ils ont en commun et où travaillent en équipe, avec les chercheurs du CNRS ou d'autres organismes, enseignants-chercheurs de l'enseignement supérieur, ingénieurs, techniciens et personnels administratifs. En octobre 1994, le CNRS est le premier organisme public de recherche à rejoindre le dispositif contractuel déjà existant entre les établissements d'enseignement supérieur et le ministère de tutelle.

Since about thirty years, CNRS and the university education system are linked by labs, in which researchers from CNRS or other institutions, researchers-university teachers, engineers or technicians work all together. In October 1994, CNRS is the first public institution to apply the contractual disposition already existing between universities and schools and the ministry of Research.

\section{AUTEUR}

\section{BERNARD BIGOT}

Bernard Bigot, chimiste, est Haut commissaire à l'énergie atomique au CEA depuis juillet 2003. Il a auparavant exercé la fonction de directeur général de la recherche et de la technologie au ministère, de directeur de l'École normale supérieure de Lyon et de directeur de cabinet du Claudie Haigneré, ministre déléguée à la Recherche et aux Nouvelles technologies (2002-2003). 